\title{
Precipitation Science: Observations, Retrievals, and Modeling
}

\author{
Francisco J. Tapiador, ${ }^{1}$ Sahra Kacimi, ${ }^{2}$ Manuel de Castro, ${ }^{1}$ Vincenzo Levizzani, ${ }^{3}$ \\ Dimitrios Katsanos, ${ }^{4}$ and Eduardo García-Ortega ${ }^{5}$
}

\author{
${ }^{1}$ Universidad de Castilla-La Mancha (UCLM), Instituto de Ciencias Ambientales (ICAM), 45071 Toledo, Spain \\ ${ }^{2}$ Jet Propulsion Laboratory, California Institute of Technology, Pasadena, CA 91109-8099, USA \\ ${ }^{3}$ CNR-ISAC, 40129 Bologna, Italy \\ ${ }^{4}$ Athens National Observatory, P.O. Box 20048, 11810 Athens, Greece \\ ${ }^{5}$ Atmospheric Physics Group, University of León, 24071 León, Spain
}

Correspondence should be addressed to Francisco J. Tapiador; francisco.tapiador@uclm.es

Received 19 March 2015; Accepted 19 March 2015

Copyright (c) 2015 Francisco J. Tapiador et al. This is an open access article distributed under the Creative Commons Attribution License, which permits unrestricted use, distribution, and reproduction in any medium, provided the original work is properly cited.

Precipitation science is becoming a thriving multidisciplinary area as a special interest topic for meteorology, climatology, hydrology, remote sensing, and computing science. This is not surprising since precipitation features a key component of many human activities, most notably agriculture and water resources management, making rain estimation and forecasting a relevant research topic for society and policymakers. A better knowledge of precipitation processes is instrumental to respond to increasingly pressing societal needs and to provide better scientific tools for dealing with hydrological problems.

This special issue gathers a number of contributions in precipitation science, including radar studies, artificial intelligence methods, modeling, geostatistical analyses, chemical research, satellite estimates, climate variability, data assimilation, computing science, solid precipitation studies, and microphysics. The synergisms between the many approaches are clear. Thus, for instance, surface precipitation is known to be a rough (i.e., not smooth) geophysical field and as such difficult to model. That makes precipitation the prime yardstick to gauge model performance. Therein, improvements in the precise quantification of rain using rain gauges, disdrometers, and satellites translate into better model tuning and at the end improved model validation.

The collection illustrates well the observed bloom of the studies focused on the Asian weather and climate, where a precise understanding of precipitation processes is perhaps even more important than in other geographical areas as that part of the world accounts for more than half of the planet population. Moreover, knowledge of such an important atmospheric process as the Meiyu translates into increased ability to model and simulate mesoscale systems in other parts of the planet. It is therefore worthy to pay attention to observations and simulations in that area to advance the precipitation science program: a program that could be defined as a multisource, multidisciplinary, and multinational effort to better understand precipitation physics in the context of increasing societal awareness of the consequences of ongoing global warming.

Francisco J. Tapiador Sahra Kacimi Manuel de Castro Vincenzo Levizzani Dimitrios Katsanos Eduardo García-Ortega 

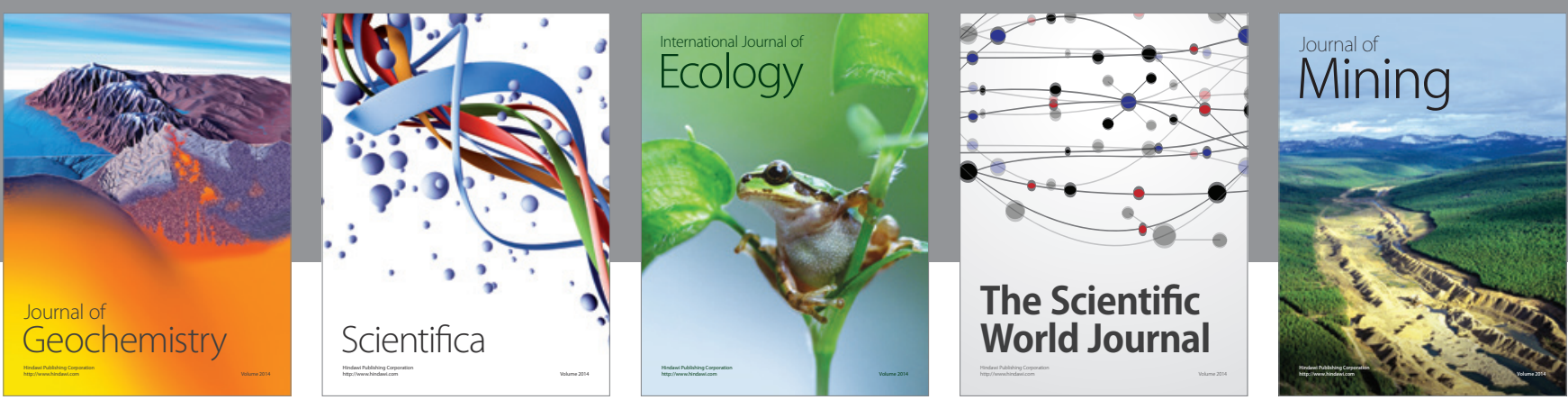

The Scientific World Journal
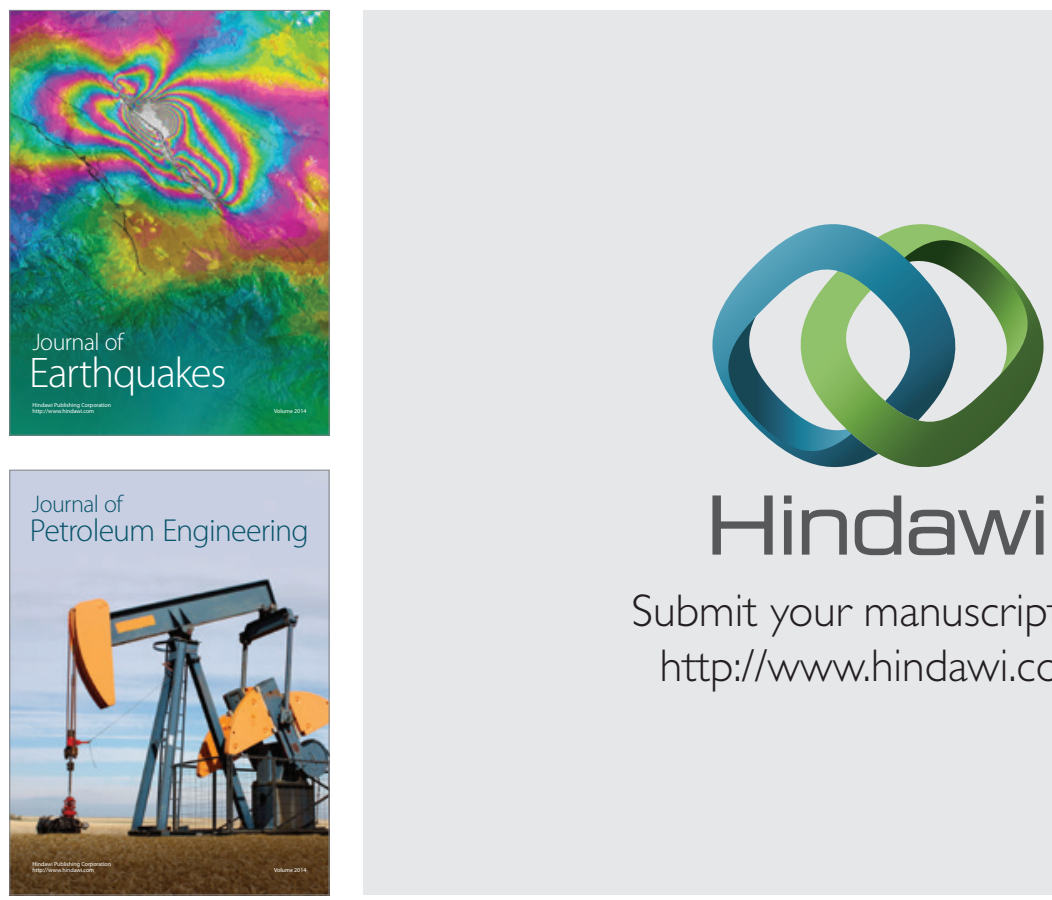

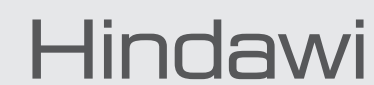

Submit your manuscripts at

http://www.hindawi.com
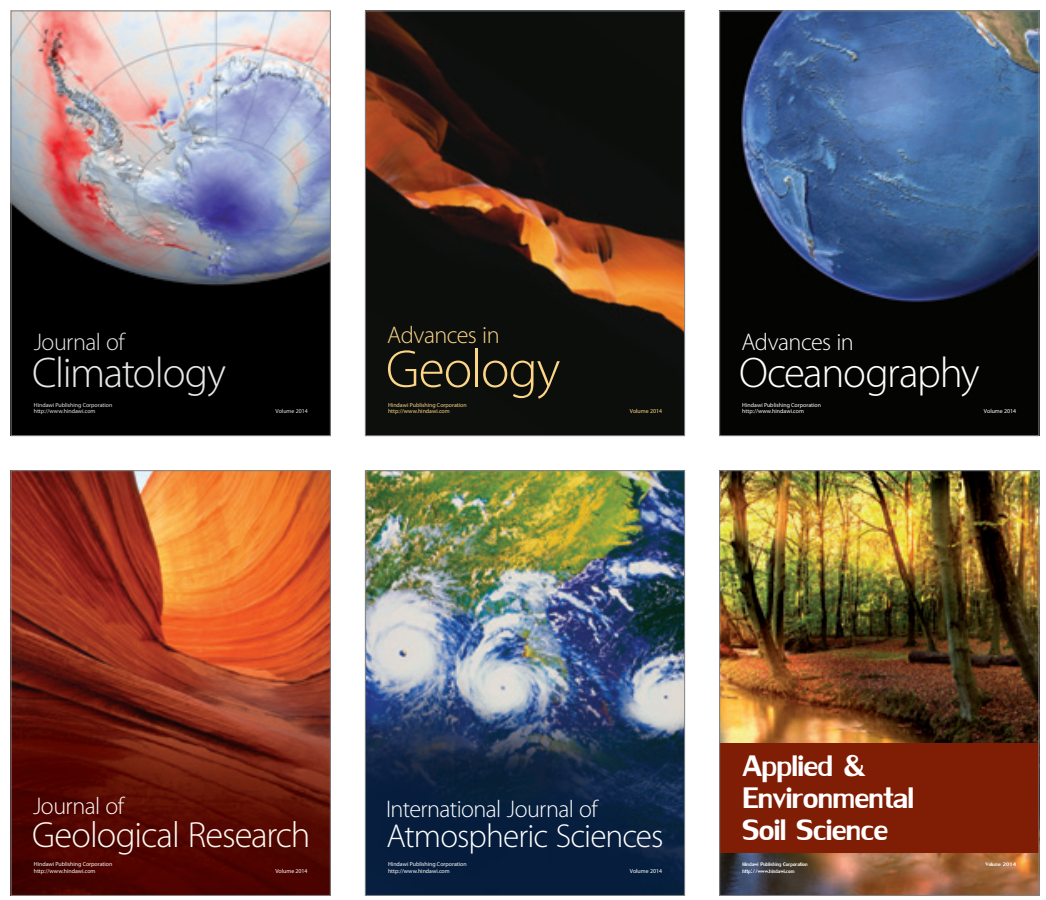
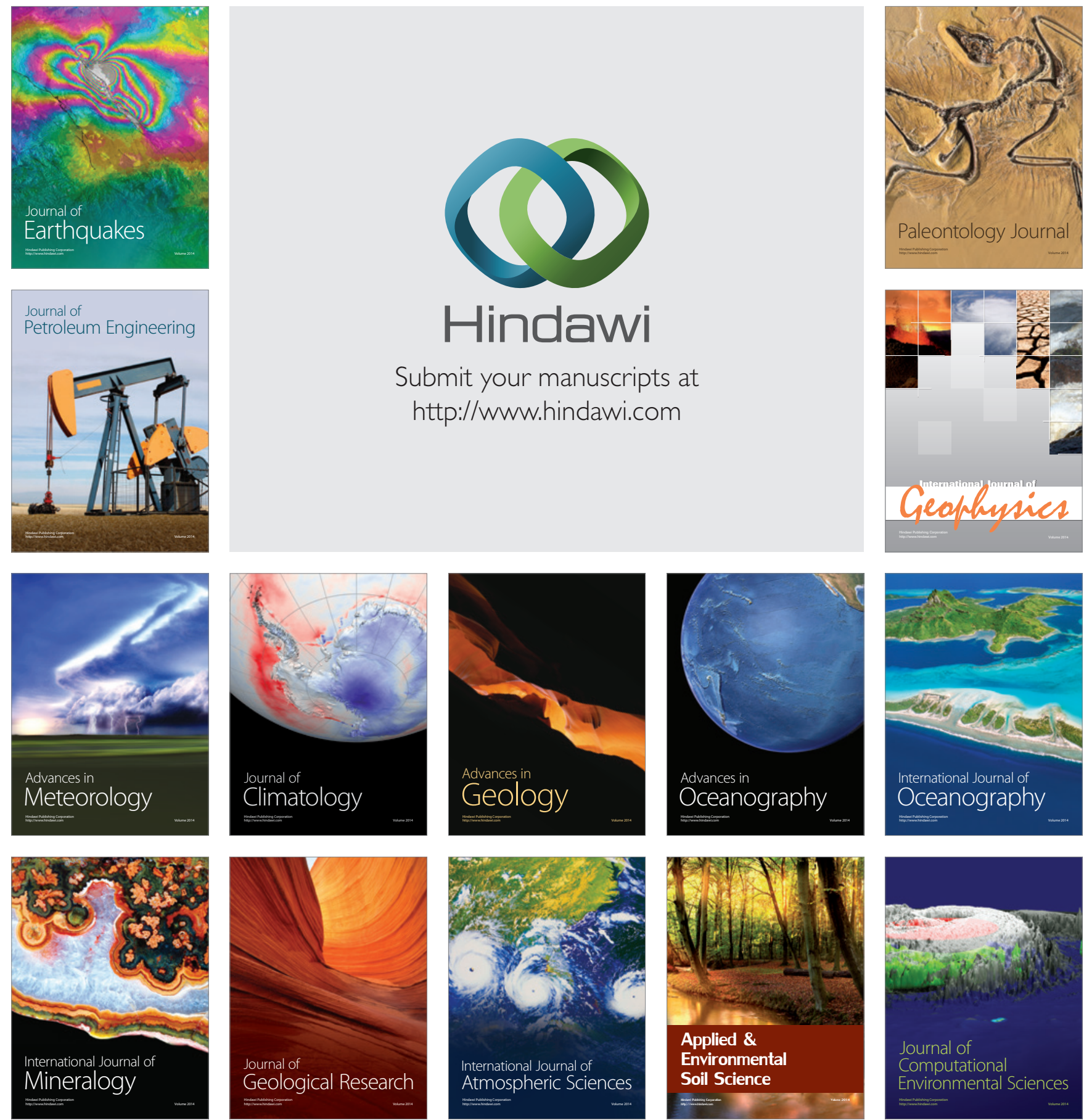